\title{
Calcium-Dependent Paired-Pulse Facilitation of Miniature EPSC Frequency Accompanies Depression of EPSCs at Hippocampal Synapses in Culture
}

\author{
Dana D. Cummings, ${ }^{1}$ Karen S. Wilcox, ${ }^{2}$ and Marc A. Dichter ${ }^{1,2,3}$ \\ ${ }^{1}$ David Mahoney Institute of Neurological Sciences and ${ }^{2}$ Departments of Neurology and ${ }^{3}$ Pharmacology, School of \\ Medicine, University of Pennsylvania, Philadelphia, Pennsylvania 19104
}

\begin{abstract}
Two forms of evoked neurotransmitter release at excitatory synapses between cultured hippocampal neurons have been described. After an action potential, it has been shown that transmitter initially is released synchronously, and this is followed by a period of "slow" asynchronous release. The "fast" synchronous component of release at these synapses has been found routinely to demonstrate paired-pulse and tetanic depression, whereas the short-term plasticity of asynchronous release has not been investigated. In the present experiments, we have used the whole-cell patch-clamp technique to record from pairs of neurons in a low-density hippocampal culture preparation to determine both the properties and underlying mechanisms of short-term plasticity of asynchronous release. It was found that an increase in miniature EPSC (mEPSC) frequency accompanied both single and multiple stimuli, and this mEPSC increase was facilitated during paired stimuli, even
\end{abstract}

when the evoked synchronous release was depressed. In addition, both the activity-dependent depression of evoked EPSCs and facilitation of asynchronous mEPSC release were dependent on $\mathrm{Ca}$ accumulation in the nerve terminal. However, the Ca-dependent mechanisms underlying these two processes could be distinguished by the differential effects of two membrane-permeant calcium chelators, BAPTA-AM and EGTA-AM. Frequency-dependent depression of evoked EP$\mathrm{SCs}$ involves a rapid rise in intraterminal $\mathrm{Ca}$, which likely triggers a process that proceeds in a Ca-independent manner, whereas the asynchronous release may be linked more directly to a sustained increase in intraterminal $\mathrm{Ca}$.

Key words: mEPSC; synaptic plasticity; BAPTA-AM; EGTAAM; hippocampal culture; paired-pulse facilitation; paired-pulse depression
A two-component model of CNS excitatory neurotransmitter release has been proposed on the basis of recent experiments at excitatory synapses in hippocampal neuronal cultures (Geppert et al., 1994; Goda and Stevens, 1994). According to this model, action potentials result in an initial "fast" synchronous release of transmitter that is accompanied by a period of "slow" asynchronous release. The fast synchronous release produces a large evoked EPSC, whereas the asynchronous release is manifested by the appearance of small, miniature EPSCs (mEPSCs) that persist for up to $500 \mathrm{msec}$ after the evoked EPSC. These two components are differentially sensitive to divalent cations, such that substitution of $\mathrm{Sr}^{2+}$ for $\mathrm{Ca}^{2+}$ can eliminate synchronous release, whereas asynchronous release is unaffected (Goda and Stevens, 1994). In addition, cultured hippocampal neurons obtained from "knockout" mice lacking synaptotagmin I retain only the asynchronous form of neurotransmitter release (Geppert et al., 1994). The short-term plasticity of the fast synchronous component of neurotransmitter release at hippocampal synapses has been examined both in culture (Forsythe and Clements, 1990; Wilcox et al., 1994; Mennerick and Zorumski, 1995) and slice preparations (Creager

\footnotetext{
Received April 29, 1996; revised June 3, 1996; accepted June 5, 1996.

This work was supported by National Institute of Neurological Disorders and Stroke Grant NS-24260 to M.A.D. D.D.C. was supported by the National Institute of General Medical Sciences Medical Scientist Training Program. We thank Margaret Price and Kay Cherian for preparation and maintenance of the tissue cultures and Gloster Aaron for help with mEPSC experiments.

Correspondence should be addressed to Dr. Marc A. Dichter, Department of Neurology, Hospital of the University of Pennsylvania, 3400 Spruce Street, Philadelphia, PA 19104.

Copyright (C) 1996 Society for Neuroscience $0270-6474 / 96 / 165312-12 \$ 05.00 / 0$
}

et al., 1980; McNaughton, 1980; Griffith, 1990; Stevens and Wang, 1995). However, to the best of our knowledge, short-term synaptic plasticity of asynchronous release has not been investigated. Therefore, at model CNS synapses, the relationship between frequency-dependent short-term plasticity of evoked synchronous and asynchronous neurotransmitter remains to be determined.

The central aims of the experiments described here are to determine whether synchronous and asynchronous release of neurotransmitter display discordant or similar frequency-dependent short-term plasticity and whether the short-term plasticity of synchronous and asynchronous release of neurotransmitter shows a similar dependence on the activity-dependent changes in $\mathrm{Ca}^{2+}$ concentration in the nerve terminal.

The experiments described here show that there is a clear discordance between the paired-pulse plasticity of evoked synchronous and asynchronous release. At interstimulus intervals that allow the first asynchronous release event to decay to baseline, paired-pulse facilitation of mEPSC frequency occurs while evoked EPSCs show paired-pulse depression. Frequencydependent depression of EPSCs and the increase in mEPSC frequency can also be discriminated on the basis of their differential dependence on calcium entry and accumulation in the nerve terminal. Both tetanic depression of EPSCs and the accompanying mEPSC frequency increase are dependent on $\mathrm{Ca}^{2+}$ entry in the nerve terminal. However, although depression depends on a transient increase in $\mathrm{Ca}^{2+}$ in the nerve terminal, the accompanying mEPSC frequency increase seems to depend on $\mathrm{Ca}^{2+}$ accumulation in the nerve terminal. 
Some of the results presented here have been presented previously in abstract form (Cummings and Dichter, 1994).

\section{MATERIALS AND METHODS}

\section{Tissue culture}

Low-density primary dissociated cultures of hippocampal neurons were prepared as described previously (Wilcox and Dichter, 1994; Wilcox et al., 1994). Neurons were used at 14-35 d in vitro.

\section{Electrophysiological recordings}

Whole-cell patch-clamp recordings (Hamill et al., 1981) were obtained readily from pairs of neurons maintained in culture for 2-5 weeks. Relatively isolated pairs of neurons were visualized at $320 \times$ magnification on a Zeiss inverted-light microscope. Low-impedance borosilicate glass electrodes (2-6 M $\Omega$ ) were used for both neurons in a pair. The presynaptic recording initially was obtained in voltage-clamp mode before switching to current-clamp mode on either an Axopatch 200A or Dagan 8900 amplifier. The postsynaptic neuron was recorded in voltageclamp mode. The recorded cells were perfused locally at $0.5-1.0 \mathrm{ml} / \mathrm{min}$ with a macroperfusion tube inlet and outlet from a peristaltic pump. All experiments were conducted at room temperature $\left(22-25^{\circ} \mathrm{C}\right)$. To monitor stationarity of the recording characteristics, leak resistance was measured periodically during the recording and ranged from 250 to $1 \mathrm{G} \Omega$ for a given cell. Leak current monitored on either amplifier in voltage-clamp mode ranged from 20 to $500 \mathrm{pA}$. Collection of data for each recording was terminated if the postsynaptic recording showed a significant change in leak resistance. Fast and slow capacitance and series resistance compensation were performed in whole-cell mode. Recording data were acquired at $10 \mathrm{kHz}$ in real time on videotape with a VR10A digital data recorder (Instrutech) and VCR. Presynaptic current injections and data acquisition of evoked responses and accompanying spontaneous currents were provided in real time with pClamp Clampex software and a TL-1 DMA data acquisition interface (Axon Instruments, Foster City, CA) on a 386 PC.

\section{Recording solutions}

The extracellular HEPES-buffered saline (HBS) solution contained (in mM): $\mathrm{NaCl} 140, \mathrm{KCl} 3$, HEPES-NaOH buffer 10, glucose $10, \mathrm{CaCl}_{2} 2$ or 4, and $\mathrm{MgCl}_{2}$ 1. Experiments were conducted in HBS 4:1 $\left(\left[\mathrm{Ca}^{2+}\right] /\left[\mathrm{Mg}^{2+}\right]\right)$ instead of HBS $2: 1$ in some cases to improve ease of recording and to suppress other synapses in the neighborhood that could have contaminated our responses. Because the results in the two extracellular solutions were qualitatively similar, we have combined results except in experiments in which a distinction was noted and stated. During some experiments extracellular $\mathrm{Ca}^{2+} / \mathrm{Mg}^{2+}$ ratio was varied to alter release properties while maintaining the total divalent concentration constant (Frankenhaeuser and Hodgkin, 1957).

AMPA receptor-mediated responses were chosen for analysis because their rapid onset and decay allow reliable postsynaptic detection of high-frequency neurotransmitter release from the presynaptic nerve terminal. The long time course of NMDA receptor-mediated events made resolution of individual spontaneous mEPSCs difficult. In most experiments $50-100 \mu \mathrm{m}$ of 2-amino-5-phosphonopentanoic acid (APV) was added to the perfusion solution, and the postsynaptic cell was held in voltage clamp at $-80 \mathrm{mV}$ to block NMDA receptor-mediated postsynaptic currents. To ensure that frequency-dependent depression of EPSCs persisted under conditions in which NMDA receptor-mediated associative plasticity mechanisms might be active (Malenka and Nicoll, 1993), we also conducted experiments in extracellular solutions that would permit mixed NMDA/AMPA receptor-mediated responses (1-3 $\mu \mathrm{m}$ of glycine, 0 $\mathrm{mM} \mathrm{Mg}^{2+}$, and $20 \mu \mathrm{m}$ of bicuculline). To test whether frequencydependent depression of EPSCs occurred independently of AMPA receptor activation, experiments were also conducted in extracellular solutions that would permit pure NMDA receptor-mediated postsynaptic responses $[10 \mu \mathrm{m}$ of 6-cyano-7-nitroquinoxaline-2,3-dione (CNQX), 3 $\mu \mathrm{m}$ of glycine, $0 \mathrm{~mm} \mathrm{Mg}^{2+}$, and $20 \mu \mathrm{m}$ of bicuculline]. In most experiments, bicuculline methiodide $(10-20 \mu \mathrm{m})$ was included to block inhibitory postsynaptic responses.

The whole-cell intracellular or internal pipette solution contained (in $\mathrm{mM}$ ): potassium gluconate 130-140 and $\mathrm{KCl} 5-10$, HEPES 10, EGTA 1, $\mathrm{CaCl}_{2}$ 0.1-1.0, ATP- $\mathrm{Mg}^{2+} 2.5$, and glucose 10. The osmolarities of the intracellular and extracellular solution ranged from 285 to 295 and 295 to 310 , respectively. The $\mathrm{pH}$ of both solutions was adjusted to 7.3-7.4. Previous experiments in our preparation (Wilcox and Dichter, 1994;
Wilcox et al., 1994; Maki et al., 1995) and our results presented here were relatively insensitive to the extent of $\mathrm{Ca}^{2+}$ buffering resulting from our choice of $\mathrm{Ca}^{2+} /$ EGTA concentration ratio in our intracellular solution, suggesting that the intracellular solution in a presynaptic neuron is unlikely to be affecting the calcium homeostasis of nerve terminals directly. Cadmium $(250-500 \mu \mathrm{m})$ was added to extracellular $\mathrm{Ca}^{2+}$ in some experiments to block evoked synaptic transmission. CNQX $(10 \mu \mathrm{m})$ was used to block glutamatergic AMPA-receptor activation to check for effective perfusion of solutions over the neurons of interest. EGTA-AM and BAPTA-AM (E-1219 and B-1205; Molecular Probes, Eugene, OR), membrane-permeant forms of EGTA and BAPTA, were perfused in the extracellular solution to chelate presynaptic intracellular calcium. EGTA-AM and BAPTA-AM were dissolved in $0.5 \%$ dimethyl sulfoxide (DMSO; Sigma, St. Louis, MO).

\section{Analysis}

In some experiments evoked events were acquired by the Clampex module of pClamp and analyzed in a semiautomated manner in the Clampfit module. Most evoked events and all mEPSCs were acquired from videotape playback in a semiautomated mode in the Fetchex module.

An important issue when analyzing synaptic plasticity is determining whether the changes occur at the presynaptic or the postsynaptic level. One critical tool for localizing plasticity is the analysis of spontaneous mPSCs. mPSCs represent the postsynaptic responses to the quantal release of vesicular packets of neurotransmitter. According to the quantal hypothesis, changes in the amplitudes of mPSCs reflect a postsynaptic mechanism underlying a change in synaptic efficacy (Fatt and Katz, 1952; del Castillo and Katz, 1954) (for review, see Katz, 1969; Stevens, 1993). Presynaptic mechanisms result in no change in the amplitude of the mPSCs but may cause a change in the frequency of mPSCs detected in the postsynaptic cell. Our relatively isolated pairs of monosynaptically connected excitatory neurons in low-density hippocampal culture satisfy important criteria for using a quantal analysis for the determination of the locus of synaptic plasticity (Korn and Faber, 1991), including the following: (1) verifiable stationarity of presynaptic stimulus provided by whole-cell monitoring of presynaptic somatic action potential induced by current injection, (2) high signal-to-noise ratio necessary to resolve spontaneous miniature excitatory postsynaptic current (mEPSC) amplitudes and time of occurrences, and (3) evoked EPSCs and mEPSCs arising from the same presynaptic neuron.

In our experiments, amplitudes of mEPSCs were examined by constructing cumulative probability histograms with event amplitude on the abscissa and relative frequency on the ordinate. The amplitudes of mEPSCs were not measured until the evoked EPSC had returned to baseline. Only mEPSC amplitudes falling below a predetermined threshold that was at least two times that of stable baseline noise were acquired. Populations of mEPSCs before and after stimulation of evoked events were examined to determine differences with the nonparametric Kolmogorov-Smirnov statistic (Van der Kloot, 1991) by the use of Statistica for the Macintosh (Statsoft, Oklahoma City, OK). This statistic provides a measure of the relative dispersion between two cumulative probability distributions. Values of $p<0.05$ were chosen as cutoff values for detecting a significant difference between populations. For some recordings, total charge, instead of event amplitude, was used for the analysis to reduce the error attributable to noise. The power of the KolmogorovSmirnov Test to detect a change in amplitude distribution is dependent on the number of samples in the control and test conditions and the properties of the event amplitude distribution (Siegel, 1956). Therefore, we used the distribution of amplitude events under control conditions to estimate a threshold for detection of an amplitude distribution change that would result from a pure postsynaptic mechanism for each recording. A postsynaptic change was simulated by multiplying the total charge or amplitude of all of the events obtained in control conditions by a constant of 1.05-1.3, corresponding to mean event amplitude or charge increases of $5-30 \%$. The threshold for detection of a change in event peak amplitude distribution consistent with a postsynaptic mechanism of action was estimated by iteratively shifting the control distribution for each recording by increasing percentages of the mean until a significant difference $(p<0.05)$ was detected by the Kolmogorov-Smirnov Test.

To compare the frequency of occurrence of mEPSCs between control and drug conditions, cumulative interevent interval distributions were constructed, and the Kolmogorov-Smirnov statistic was applied to detect statistically important differences between interevent interval distributions. To analyze the time course of the onset and decay of changes in 
mEPSC frequency during stimulation experiments, histograms were constructed in Excel (Microsoft) from data for the time of occurrence of each mEPSC. High-resolution histograms were plotted with CricketGraph III (Computer Associates). For some experiments, the time course of the occurrence of mEPSCs was represented with a moving bin histogram to minimize the effect of bin size selection (Rahamimoff and Yaari, 1973). It should be noted that, although throughout our results we refer to EPSCs as representing "synchronous" release, strictly speaking EPSCs may also result from asynchronous release but with a much shorter time window of

$\sim 20 \mathrm{msec}$ or less (Diamond and Jahr, 1995), as compared with the asynchronous release of mEPSCs at our excitatory synapses, which may be dispersed over $200-600 \mathrm{msec}$.

To assess the effect of $\mathrm{Ca}^{2+}$ chelation or changes in the extracellular $\mathrm{Ca}^{2+} / \mathrm{Mg}^{2+}$ ratio on frequency-dependent depression of evoked event amplitudes, the ratio of the amplitude of the second event, p2, to the amplitude of the first event, p1, was obtained for each trial in the various experimental conditions. Paired-pulse depression (PPD) was defined as $\mathrm{p} 2 / \mathrm{p} 1<1.0$. A student's two-tailed $t$ test was used to look for significant differences $(p<0.05)$ between trials in two different conditions in individual recordings. To analyze changes in the tetanic depression of evoked responses with $20 \mathrm{~Hz}, 10$ action potential stimuli, we determined the ratio of the amplitudes of EPSCs 7-10 in an arbitrarily defined "plateau region" to the amplitude of the first event, p1, in a train tetanic response for each trial of tetany. For each recording, the ratios from the plateau region were pooled by recording condition and compared by using a student's two-tailed $t$ test to detect significant differences $(p<$ 0.05 ). EPSC amplitudes for both the initial two events in tetanic responses were compared also. When data from groups of recordings were pooled to summarize results, two-tailed paired two-sample Student's $t$ test was applied to test for significant differences $(p<0.05)$.

\section{RESULTS}

\section{EPSCs show a presynaptically mediated frequency-dependent depression}

Monosynaptic EPSCs were recorded from $>100$ pairs of hippocampal neurons. Before performing experiments to analyze short-term plasticity behavior of asynchronous release of excitatory synapses, we confirmed that these synapses showed similar PPD to what had been reported previously (Forsythe and Clements, 1990; Wilcox et al., 1994; Mennerick and Zorumski, 1995). At hippocampal synapses in culture, essentially every pair tested showed PPD in $[\mathrm{Ca}]_{\mathrm{o}}=2$ or $4 \mathrm{~mm}$. Depression occurred at the shortest interstimulus interval (ISI) tested, $50 \mathrm{msec}$, and was seen at ISIs of up to $8 \mathrm{sec}$ (data not shown). Similar PPD was seen with pure AMPA receptor-mediated EPSCs, pure NMDA receptormediated EPSCs, and mixed AMPA/NMDA receptor-mediated EPSCs at interstimulus intervals from $250 \mathrm{msec}$ to $4 \mathrm{sec}(n=8)$.

The tetanic behavior and the locus of synaptic plasticity of synchronous EPSCs was determined because some of the experiments investigating the short-term plasticity of asynchronous release of neurotransmitter used tetanic stimulation to attempt to accentuate the asynchronous release. These experiments were important to allow comparison of the short-term plasticity of synchronous release of EPSCs and asynchronous release of mEPSCs. The tetanic behavior of EPSCs was examined with stimuli composed of brief trains of 10 action potentials at 2, 10, and 20 $\mathrm{Hz}$, simulating the kind of presynaptic action potential bursts that may occur in the hippocampus and neocortex (Connors and Gutnick, 1990; Traub and Miles, 1991). Each of these trains resulted in tetanic depression of the EPSCs, both during the train $(n=15$ at $20 \mathrm{~Hz})$ as well as $1 \mathrm{sec}$ after the train $(n=8)$. In conditions permitting both AMPA/NMDA mixed responses, tetanic and post-tetanic depression persisted $(n=5)$. Demonstrating that activation of CNQX-sensitive AMPA receptors was not required, tetanic depression of pure NMDA receptor-mediated EPSCs also occurred with low-frequency action potential trains (2 $\mathrm{Hz}$ for 10 events) $(n=2)$. The persistence of tetanic depression under conditions allowing AMPA, NMDA, or mixed receptormediated EPSCs is consistent with a presynaptic mechanism of tetanic depression.

To provide another way of assessing the importance of presynaptic mechanisms in accounting for tetanic depression, experiments were conducted while varying the $\mathrm{Ca}^{2+} / \mathrm{Mg}^{2+}$ ratio and maintaining a constant total divalent cation concentration during paired-pulse stimulation (Frankenhaeuser and Hodgkin, 1957; Mallart and Martin, 1968). Within a $20 \mathrm{~Hz}$ train, PPD between the first two responses was attenuated in four of six recordings $(p<$ 0.05 ), and tetanic depression of the events in the plateau region, $\mathrm{p} 7, \mathrm{p} 8, \mathrm{p} 9$, and $\mathrm{p} 10$, was also attenuated in six of six recordings $(p<0.05)$ in HBS 1:2 versus HBS 2:1 $(n=6$; Fig. 1$)$. In contrast to experiments at the neuromuscular junction (Mallart and Martin, 1968; Zucker, 1989), lowering the $\mathrm{Ca}^{2+} / \mathrm{Mg}^{2+}$ ratio never converted tetanic depression to tetanic facilitation.

Although the above data provide indirect evidence that presynaptic mechanisms mediate tetanic depression of EPSCs, the ability to resolve mEPSCs, reflecting the quantal amplitude, before and after tetanic stimulation allows for a direct assessment of the relative role of presynaptic and postsynaptic mechanisms. According to the quantal hypothesis, tetanic depression would be attributable to a presynaptic mechanism if there were no change in the amplitude distribution of mEPSCs after stimulation. A postsynaptic mechanism underlying depression would be accompanied by a decline in the amplitudes of mEPSCs after the stimulation (Korn and Faber, 1991; Van der Kloot, 1991).

Consistent with previous results at excitatory hippocampal synapses (Mennerick and Zorumski, 1995), no significant change in mEPSC amplitude distributions, occurring up to $10 \mathrm{sec}$ before and $1 \mathrm{sec}$ after paired pulses (1 sec ISI), was detected in six analyzed recordings showing PPD. Tetanic depression of EPSCs at $20 \mathrm{~Hz}$ resulted in no significant decrease in the mEPSC amplitude in six of six recordings (Fig. 2; although one pair exhibited a small but significant increase in the mean amplitude of mEPSCs) (see Table 1 for details of each of the analyzed recordings). Therefore, PPD and tetanic depression of EPSCs at hippocampal synapses in culture could be accounted for by presynaptic mechanisms.

\section{Paired-pulse plasticity of mEPSCs accompanies PPD of EPSCs}

As mentioned earlier, even after a single action potential, mEPSC frequency increases (Goda and Stevens, 1994; Mennerick and Zorumski, 1995). During our experiments examining the tetanic behavior of excitatory synapses, we noted that mEPSC frequency also increased during the train and that the enhanced release of mEPSCs (asynchronous release) could last for hundreds of milliseconds after the last stimulus, even when the final evoked EPSC amplitudes were a small fraction of the first EPSC in the train (Fig. 3). Thus, it seemed that there could be a differential plasticity between synchronous and asynchronous release during tetanic stimuli. This increase in mEPSC frequency accompanying tetanic stimulation was consistent with the results of experiments at the frog neuromuscular junction (Zengel and Sosa, 1994) and at the avian nucleus magnocellularis synapse (Zhang and Trussell, 1994) showing a tetanic increase in mEPSC frequency.

To examine the plasticity of both forms of release in detail, we examined the paired-pulse behavior of asynchronous neurotransmitter release with action potentials delivered at interstimulus intervals that allowed the asynchronous release resulting from the first action potential to decay back to baseline before the second action potential occurred. Even with paired-pulse interstimulus 
A

HBS $2 / 1$

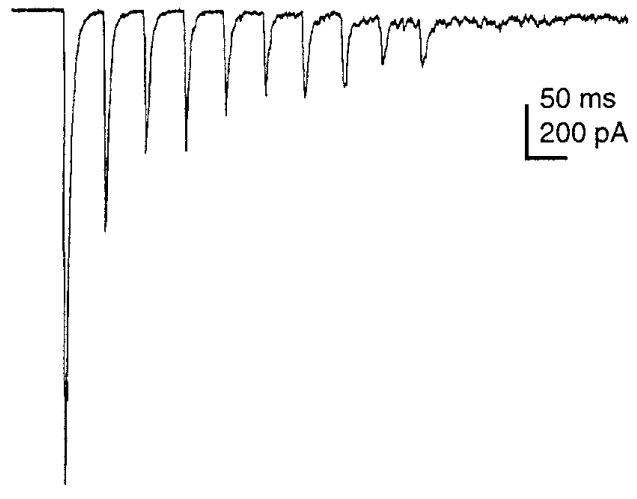

HBS $1 / 2$
B

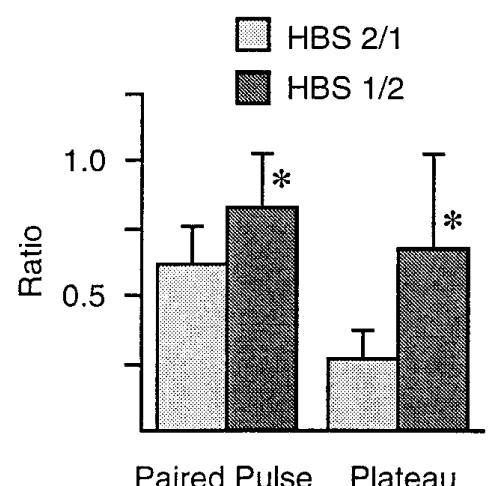

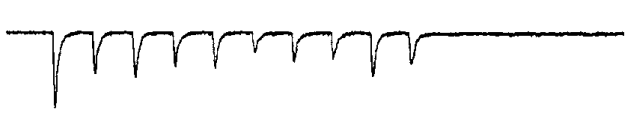

Figure 1. Varying $\left[\mathrm{Ca}^{2+}\right] /\left[\mathrm{Mg}^{2+}\right]$ ratio modulates tetanic depression with $20 \mathrm{~Hz}$ stimulation. $A$, Representative traces from a single recording in HBS $2: 1$ and HBS $1: 2$ show that lowering the $\left[\mathrm{Ca}^{2+}\right] /\left[\mathrm{Mg}^{2+}\right]$ ratio decreases the amplitude of evoked EPSCs and attenuates the relative amount of depression. $B$, The effect of varying the $\left[\mathrm{Ca}^{2+}\right] /\left[\mathrm{Mg}^{2+}\right]$ ratio on tetanic depression was determined by analyzing both the paired-pulse ratio and plateau ratio (see Materials and Methods) for EPSCs induced by $20 \mathrm{~Hz}$ trains of presynaptic action potentials. Lowering the $\left[\mathrm{Ca}^{2+}\right] /\left[\mathrm{Mg}^{2+}\right] \mathrm{ratio}$ resulted in a significant attenuation of paired-pulse and tetanic depression as assessed by plateau ratio $(p<0.05$ for two-tailed paired two-sample Student's $t$ test; $n=6)$. ${ }^{*}$, Significant difference.

intervals of $500 \mathrm{msec}$, the asynchronous release of neurotransmitter failed to decay to baseline before the second action potential $(n=3$; Fig. $4 A)$. However with a $1 \mathrm{sec}$ interstimulus interval, the asynchronous release resulting from the first action potential decayed to baseline before the second action potential in all cases $(n=5)$ with a mean time constant $( \pm$ SD) of $190 \pm 70 \mathrm{msec}$, similar to the time course described previously at hippocampal synapses (Fig. 4B,C; Goda and Stevens, 1994; Mennerick and Zorumski, 1995). When mEPSCs were examined after the second, depressed, EPSC of a pair, mEPSC frequency was actually potentiated over that occurring after the first evoked EPSC of the pair (Fig. 4). The potentiated frequency decayed to baseline with a mean time constant $( \pm \mathrm{SD})$ of $210 \pm 60 \mathrm{msec}$. Therefore, evoked

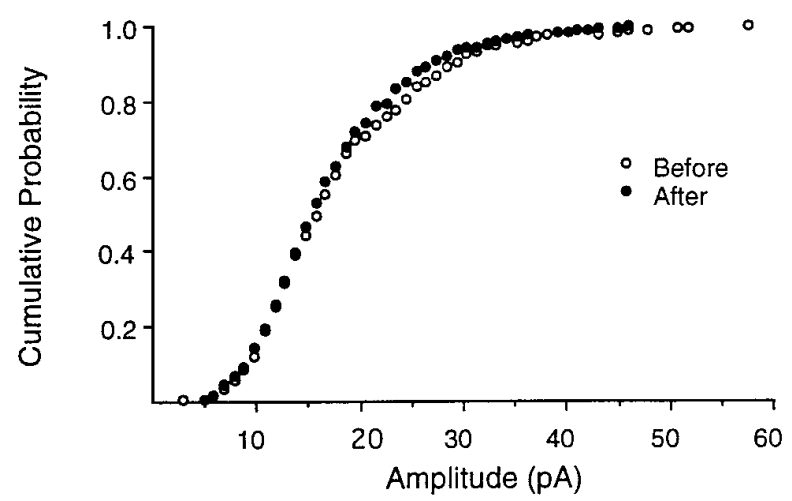

Figure 2. No change in the distribution of mEPSC amplitudes occurs with tetanic depression of EPSCs. For a representative recording, cumulative probability distributions of mEPSC amplitudes up to $10 \mathrm{sec}$ before and $1 \mathrm{sec}$ after tetanic stimulation $(20 \mathrm{~Hz}, 10$ event stimulation) show no significant difference (Kolmogorov-Smirnov nonparametric statistic). Similar results were obtained for a total of six recordings. and asynchronous neurotransmitter release at our hippocampal excitatory synapses display a discordant short-term plasticity, with paired-pulse facilitation of mEPSC frequency accompanying PPD of EPSCs.

\section{mEPSC frequency increase depends on $\mathrm{Ca}^{2+}$ entry and accumulation in the nerve terminal}

Given the above results demonstrating a discordance of the behavior between evoked synchronous and evoked asynchronous

Table 1. Effect of paired-pulse and tetanic stimulation on mEPSC peak amplitude

\begin{tabular}{lllll}
\multicolumn{5}{c}{ mEPSC peak amplitude $(\mathrm{pA})$} \\
\cline { 2 - 5 } & $\begin{array}{l}\text { Cell } \\
\text { number }\end{array}$ & $\begin{array}{l}\text { Before } \\
\text { stimulation }\end{array}$ & $\begin{array}{l}\text { After } \\
\text { stimulation }\end{array}$ & Significance \\
\hline Paired pulse & 1 & $17.6 \pm 6.9$ & $18.0 \pm 6.7$ & NS \\
& 2 & $21.2 \pm 12.5$ & $17.9 \pm 6.1$ & NS \\
& 3 & $54.1 \pm 41.9$ & $49.9 \pm 27.7$ & NS \\
& 4 & $42.8 \pm 22.0$ & $36.8 \pm 20.1$ & NS \\
& 5 & $20.2 \pm 8.6$ & $20.2 \pm 5.6$ & NS \\
Tetanic & 6 & $22.9 \pm 8.8$ & $22.6 \pm 8.8$ & NS \\
& 1 & $21.1 \pm 9.8$ & $23.6 \pm 10.8$ & $p<0.05^{*}$ \\
& 2 & $18.2 \pm 8.6$ & $17.3 \pm 7.6$ & NS \\
& 3 & $42.0 \pm 22.8$ & $41.0 \pm 14.4$ & NS \\
& 4 & $26.6 \pm 17.6$ & $27.7 \pm 11.7$ & NS \\
& 5 & $15.1 \pm 8.3$ & $14.9 \pm 8.5$ & NS \\
& 6 & $29.5 \pm 16.2$ & $31.4 \pm 19.0$ & NS
\end{tabular}

*Note mEPSCs are slightly larger after stimulation, despite depression of evoked responses.

NS, Not significant; paired-pulse stimulation at $1 \mathrm{sec}$ ISI.

Tetanic stimulation pattern $=10$ action potentials at $20 \mathrm{~Hz}$. 

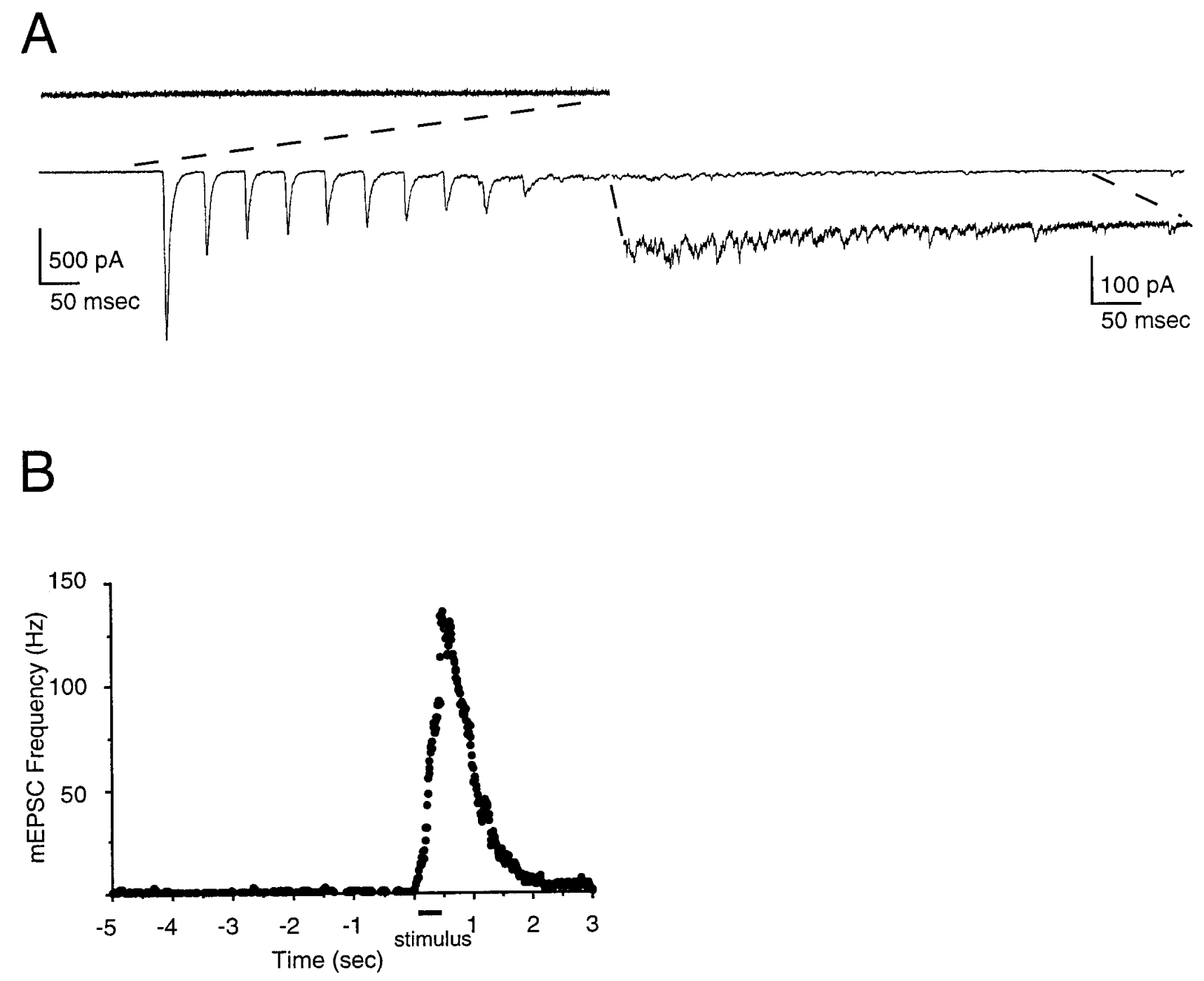

Figure 3. mEPSC frequency increase accompanies tetanic depression with $20 \mathrm{~Hz}$ stimulation. $A$, Evoked EPSCs in a $20 \mathrm{~Hz}$ train with accompanying magnified regions showing an absence of mEPSCs in the period from 700 to $80 \mathrm{msec}$ before the train and a dramatic increase in the frequency of mEPSCs in the period from 80 to $700 \mathrm{msec}$ after the train. $B$, For the recording in $A$, a moving bin histogram for mEPSC occurrence times shows a steady increase in the mEPSC frequency during the train and a post-train decay in mEPSC frequency with an estimated time constant of $530 \mathrm{msec}$. Data for the histogram were obtained by averaging the occurrence time-binned histograms for 36 trials. A moving bin histogram was constructed with $50 \mathrm{msec}$ bins formed by shifting the bin by $10 \mathrm{msec}$ for each point to minimize the effects of binning on the time course of the mEPSC frequency increase (Rahamimoff and Yaari, 1973). Bar indicates time of $20 \mathrm{~Hz}$ stimulus $(0-450 \mathrm{msec})$. The tetanic increase in mEPSC frequency accompanying tetanic depression was present in all nine excitatory synapses for which mEPSC occurrence time-binned histograms were generated.

neurotransmitter release, we began to investigate the mechanisms that might explain this behavior. We attempted to determine the role of extracellular $\mathrm{Ca}^{2+}, \mathrm{Ca}^{2+}$ entry, and $\mathrm{Ca}^{2+}$ accumulation in the nerve terminal in the short-term plasticity of mEPSC frequency and EPSC amplitude. The mEPSC frequency increase accompanying a $20 \mathrm{~Hz}$ train stimulus was extremely sensitive to the $\mathrm{Ca}^{2+} / \mathrm{Mg}^{2+}$ ratio. Changing the $\mathrm{Ca}^{2+} / \mathrm{Mg}^{2+}$ ratio from $2: 1$ to 1:2 or from $4: 1$ to $1: 4$ caused a drastic reduction in the mEPSC frequency increase accompanying frequency-dependent depression of EPSCs $\left(n=4\right.$; Fig. 5). Whereas lowering the $\mathrm{Ca}^{2+} / \mathrm{Mg}^{2+}$ ratio converts tetanic depression to tetanic facilitation at neuromuscular junctions (Mallart and Martin, 1968; Zucker, 1989), tetanic depression of EPSCs at hippocampal synapses persisted, although in an attenuated form (see Fig. 1 and above results).

To address more directly the role of $\mathrm{Ca}^{2+}$ entry in the terminal, the $\mathrm{Ca}^{2+}$ channel blocker cadmium was added to the extracellular solution. With presynaptic stimulation of trains of 10 action potentials at $20 \mathrm{~Hz}, \mathrm{Cd}^{2+}$ blocked both the evoked events and the increase in mEPSCs accompanying synaptic activity $(n=9$; Fig. 6). To ensure that $\mathrm{Cd}^{2+}$ was not having a direct effect on postsynaptic sensitivity, its effect on the amplitude distributions of spontaneous mEPSCs in the presence of TTX was evaluated. $\mathrm{Cd}^{2+}$ produced no detectable change in the amplitude distributions of mEPSCs $(n=5$; Table 2$)$. In addition, $\mathrm{Cd}^{2+}$ produced no significant increase in the mean interevent interval of mEPSCs $(n=6)$ and even a significant decrease in the mean interevent interval of mEPSC in two recordings, indicating that transmembrane $\mathrm{Ca}^{2+}$ flux probably was not playing a major role in spontaneous release of quanta of excitatory neurotransmitter in these neurons (Table 2).

The next set of experiments was designed to determine whether 


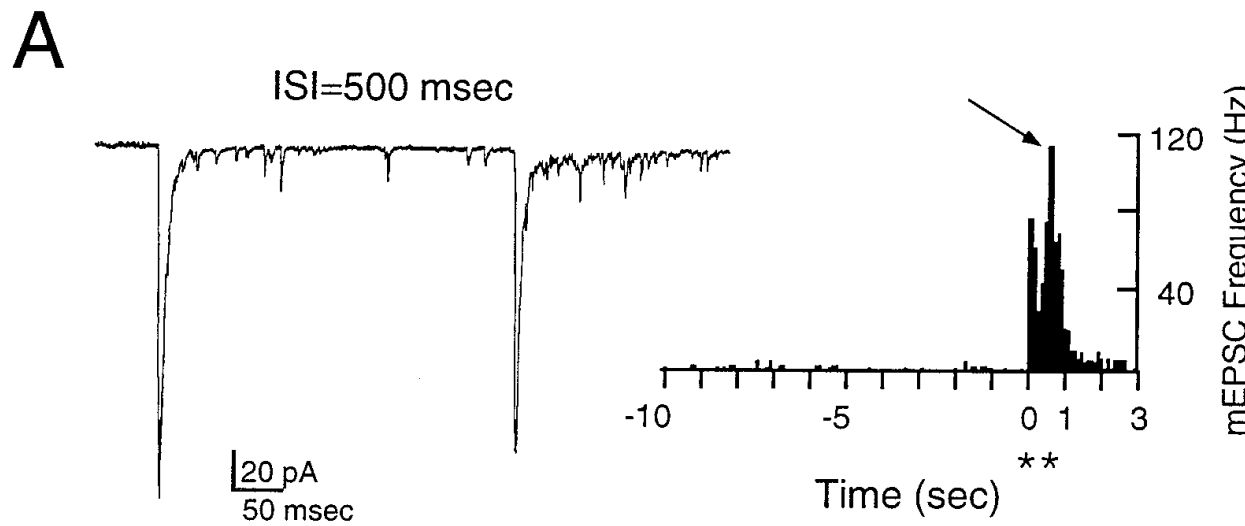

Figure 4. Paired-pulse facilitation of mEPSC frequency accompanies PPD of evoked EPSCs. $A$, Paired-pulse stimulation $($ ISI $=500 \mathrm{msec})$ causes a decrease in the amplitude of the second EPSC. When the
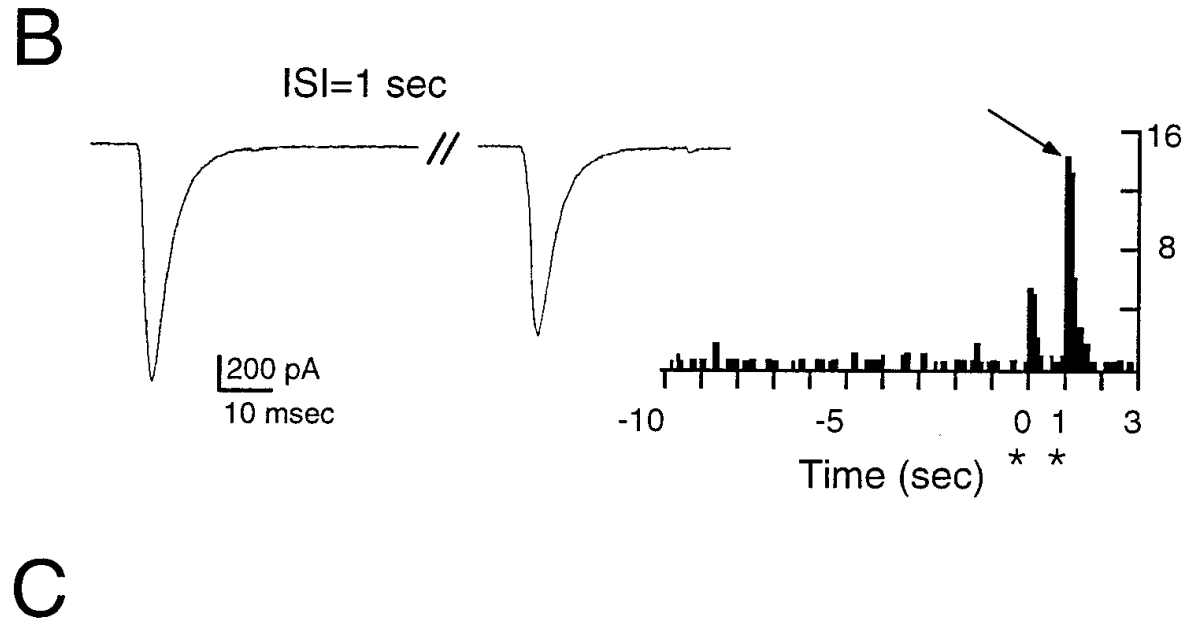

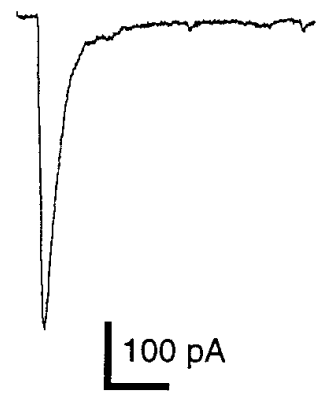

$20 \mathrm{msec}$

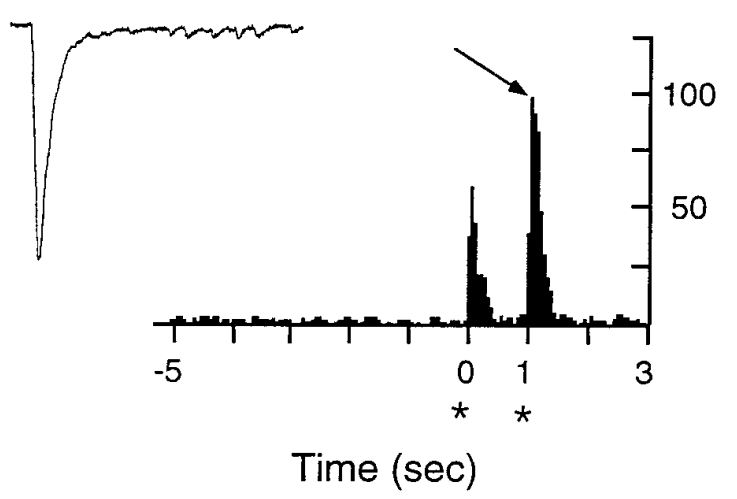
frequency of the occurrence of mEPSCs is plotted as a function of time from stimulation, it is readily apparent that not only does the frequency increase after a single EPSC, but the increase in frequency is $\underline{\text { fa- }}$ cilitated after the second EPSC (average behavior for 8 trials). Arrow indicates time of the second EPSC (p2). Increase in mEPSC frequency after the second EPSC decayed with an estimated time constant of $270 \mathrm{msec}$. The time constant was estimated from moving bin representation of mEPSC occurrence times. Bin size, $50 \mathrm{msec}$; *, time of presynaptic action potentials. $B$, Another paired recording demonstrates facilitation of mEPSC frequency accompanied by a paradoxical PPD of EPSCs after action potentials evoked with an ISI $=1 \mathrm{sec}$ (average behavior for 36 trials). Increases in mEPSC frequency after the first and second EPSC both decayed with estimated time constants of $180 \mathrm{msec}$. Paired-pulse facilitation of mEPSC frequency was observed in eight recordings that were analyzed. $C$, Another paired recording with an ISI of $1 \mathrm{sec}$ (average behavior for 13 trials) demonstrates that paired-pulse facilitation of mEPSC frequency persists even when the mEPSC frequency accompanying the first EPSC is much greater (compare mEPSC frequency increases in $B$ and $C$ ). The increase in mEPSC frequency after the first and second EPSC decayed with estimated time constants of 160 and $170 \mathrm{msec}$, respectively.

$\mathrm{Ca}^{2+}$ accumulation via transmembrane $\mathrm{Ca}^{2+}$ flux in the nerve terminal regulates the activity-dependent mEPSC frequency increase. Membrane-permeant forms of $\mathrm{Ca}^{2+}$ chelators/buffers with different kinetic properties were used to dissect the $\mathrm{Ca}^{2+}$ dependent processes involved in evoked synchronous and asynchronous release (Adler et al., 1991; Swandulla et al., 1991; Tymianski et al., 1993). Control responses to $20 \mathrm{~Hz}$ train stimulation were obtained, and extracellular solution containing $50 \mu \mathrm{m}$ of BAPTA-AM was perfused into the bath. Within 3-5 min after the start of BAPTA-AM perfusion, evoked responses were diminished, but not blocked, and frequency-dependent depression was attenuated ( $n=7$; Fig. 7). The frequency-dependent mEPSC increase accompanying train stimulation also was attenuated by application of $50 \mu \mathrm{m}$ of BAPTA-AM $(n=4)$. The attenuation of mEPSC frequency enhancement resulting from BAPTA-AM per- fusion was reversed by prolonging the duration of train stimulation, suggesting that BAPTA-AM in the nerve terminal was saturable $(n=4$; Fig. $7 D)$. To verify that the BAPTA-AM-mediated attenuation of frequency-dependent depression of EPSCs was mediated by a presynaptic mechanism, the effect of BAPTA-AM on the amplitude distribution of spontaneous mEPSCs recorded in TTX was determined ( $n=8$ cells). Consistent with a predominantly presynaptic effect, BAPTA-AM $(200 \mu \mathrm{M})$, a concentration four times that used for the experiments described above, caused no significant change in mean mEPSC amplitude in five of eight cells (Kolmogorov-Smirnov test). Two cells demonstrated a very slight but significant increase in mean mEPSC, whereas one cell showed a significant decrease in amplitude.

Because BAPTA-AM was dissolved in a final concentration of 0.25 or $0.5 \%$ DMSO and DMSO has been reported to affect 


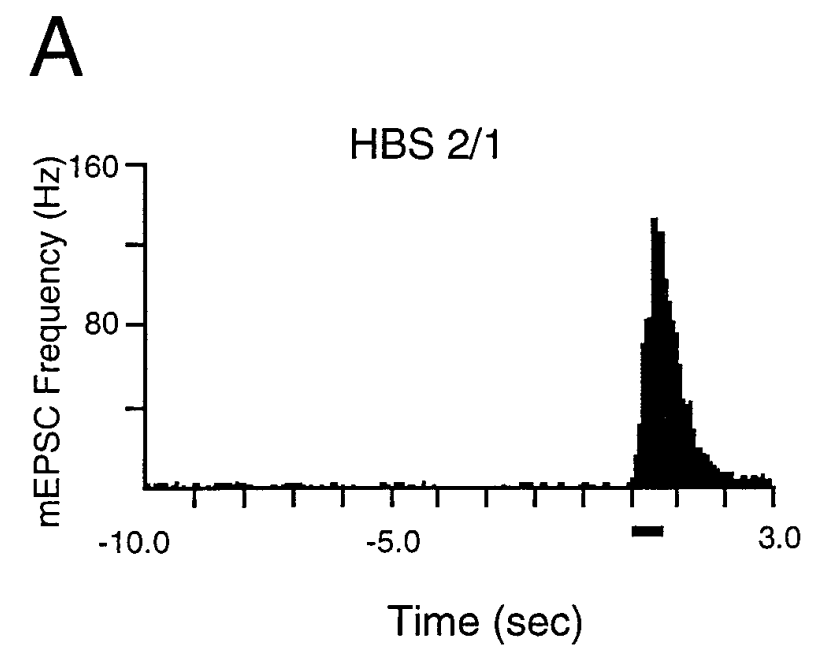

$\mathrm{B}$

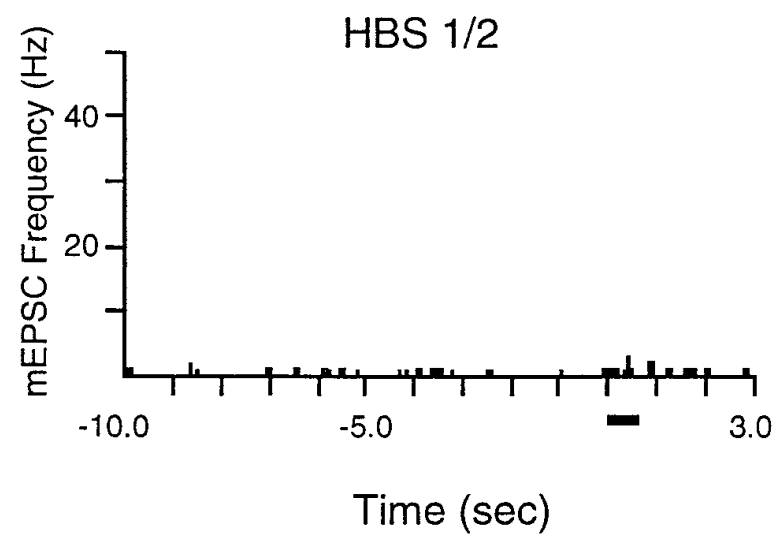

Figure 5. $\quad$ mEPSC frequency increase with $20 \mathrm{~Hz}$ stimulation depends on external $\left[\mathrm{Ca}^{2+}\right] /\left[\mathrm{Mg}^{2+}\right]$. Average occurrence time-binned histograms $($ bin $=50$ $\mathrm{msec}$ ) show times of occurrence of mEPSCs relative to train stimulation (time, $0-450 \mathrm{msec}$ ) for data collected from indicated number of trials. $A$, In HBS $2: 1$, tetanic increase in mEPSC frequency is apparent (36 trials). $B$, Changing extracellular solution to HBS $1: 2$ attenuates the mean increase in mini frequency (15 trials). Note the difference in $y$-axis scales. This recording, with attenuation of mEPSC frequency, is the same recording pictured in Figure 1 that shows a coincident persistence of tetanic depression of EPSCs in HBS 1:2.

presynaptic terminals and release properties (McLarnon et al., 1986), DMSO was added to control bath solutions in some experiments so that the control bath solutions and BAPTA-AM solutions contained equivalent concentrations of DMSO. In recordings with cells bathed in $0.25-0.5 \%$ DMSO, both tetanic depression of evoked EPSC amplitudes and the increase in mEPSC frequency accompanying a $20 \mathrm{~Hz}, 10$ event tetanic stimulus persisted $(n=6)$. In addition, DMSO $(0.5 \%)$ had no effect on spontaneous mEPSCs recorded in the presence of TTX $(n=6$ cells).

Given that BAPTA-AM interfered with both the tetanic depression of EPSCs and the mEPSC frequency increase, a calcium chelator with slower $\mathrm{Ca}^{2+}$ association kinetics was used to attempt to interfere selectively with one of the two components of release. The experiments were repeated with EGTA-AM, a parent compound of BAPTA-AM with similar $\mathrm{Ca}^{2+}$ affinity but a slower forward $\mathrm{Ca}^{2+}$ association rate constant (Adler et al., 1991; Augustine et al., 1991; Tymianski et al., 1994). EGTA-AM attenuated the increase in mEPSC frequency after a train of evoked events, but it had no effect on either the amplitude of EPSCs or the magnitude of tetanic depression ( $n=5$; Fig. 8). We conclude that the mEPSC frequency increase accompanying tetanic depression requires $\mathrm{Ca}^{2+}$ entry and accumulation in the nerve terminal. The difference between the effects of BAPTA-AM and EGTA-AM suggests that tetanic depression of EPSCs is activated by rapid transient changes in intraterminal $\mathrm{Ca}^{2+}$ concentration, whereas the increase in mEPSC frequency depends on a slow accumulation of $\mathrm{Ca}^{2+}$ in the nerve terminal.

\section{DISCUSSION}

\section{Paradoxical paired-pulse plasticity of mEPSC frequency accompanies presynaptically mediated depression of EPSCs}

Recent work suggested the possibility that CNS synapses use two forms of evoked neurotransmitter release, which involve synchronous and asynchronous release of quanta of neurotransmitter in response to a single action potential (Geppert et al., 1994; Goda and Stevens, 1994; Mennerick and Zorumski, 1995). Synchronous release has traditionally been regarded as the basic currency of information transfer within the central nervous system. As yet, there is no identified function for asynchronous release. The critical next step suggested by the previous work was the determination of whether the asynchronous release mechanism could act independently of evoked synchronous release during shortterm plasticity of excitatory synapses. Our results, which demonstrate a paradoxical paired-pulse facilitation of asynchronous neurotransmitter release accompanying paired-pulse depression of synchronous neurotransmitter release, provide the first clear evidence that these two forms of release have different responses to synaptic activation consistent with independent underlying mechanisms.

The conclusion that these model CNS synapses display a paradoxical, discordant presynaptically mediated short-term plasticity of the synchronous and asynchronous release of neurotransmitter depended on our demonstration that presynaptic mechanisms mediate both the paired-pulse facilitation of asynchronous release and the frequency-dependent depression of EPSCs. According to the quantal hypothesis, the paired-pulse facilitation of asynchronous release of mEPSCs must be dependent on a mechanism with a presynaptic site of expression (Fatt and Katz, 1952; del Castillo and Katz, 1954). In contrast, the depression of EPSC amplitude could be accounted for by a presynaptic or postsynaptic mechanism. If a postsynaptic mechanism accounted for the depression of EPSCs, then the explanation of paradoxical paired-pulse facilitation of asynchronous release accompanying paired depression of synchronous release would not require discordant presynaptic mechanisms; although a common presynaptic plasticity mechanism could produce a presynaptic enhancement of both synchronous EPSC amplitude and asynchronous release of mEPSCs, a postsynaptic mechanism of depression could mask the presynaptic enhancement of EPSC amplitude, resulting in a decrease in EPSC amplitude.

We have provided several pieces of evidence consistent with a presynaptic mechanism for frequency-dependent depression of EPSCs. First, EPSCs mediated by activation of only AMPA or 


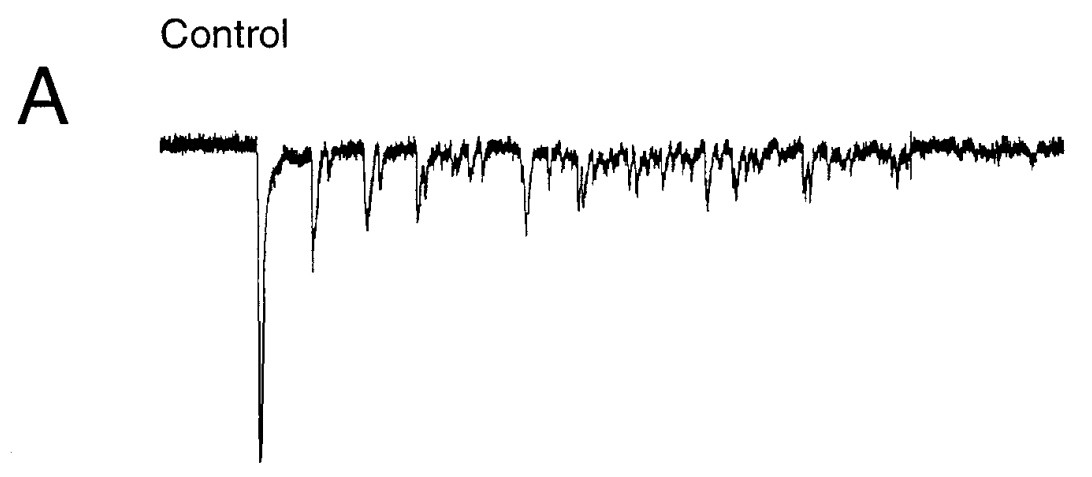

\section{$500 \mu \mathrm{M} \mathrm{Cd}$}

B
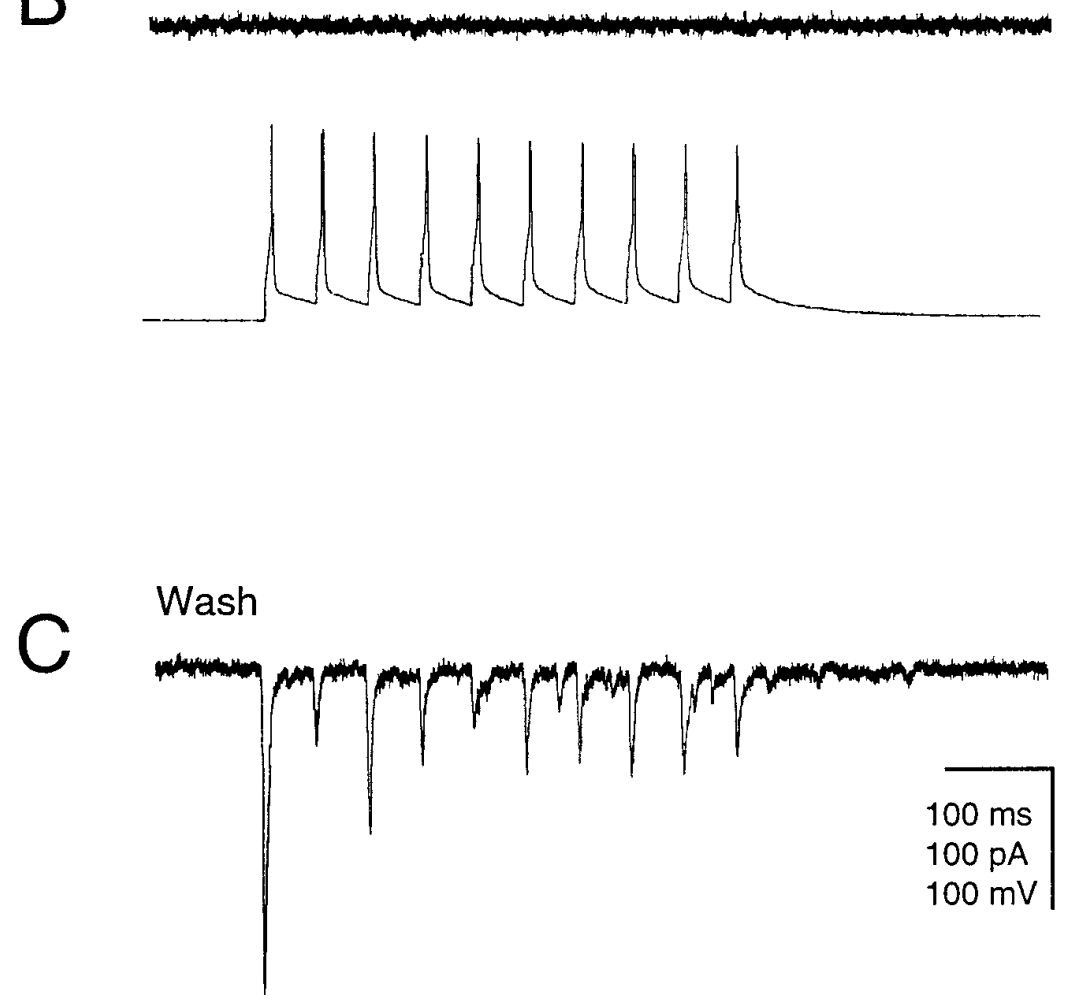

Figure 6. $\mathrm{Cd}^{2+}$ blocks both evoked release and mEPSC frequency increase with $20 \mathrm{~Hz}$ train stimulation. $A$, In HBS $2: 1$, frequency-dependent depression of EPSCs and mEPSC frequency increases are seen. $B$, In the same recording, 500 $\mu \mathrm{M} \mathrm{Cd}^{2+}$ blocks evoked release and the accompanying mEPSC frequency increase despite persistence of the presynaptic action potentials. $C$, Loss of evoked EPSCs and mEPSC frequency increase are reversible when medium is changed back to HBS 2:1. $\mathrm{Cd}^{2+}$ blocked evoked release and mEPSC frequency increase in all nine recordings examined. The effect of $\mathrm{Cd}^{2+}$ on the frequency and amplitude distribution of spontaneous mEPSCs is summarized in Table 2 .

NMDA receptors or both all displayed a comparable degree of tetanic depression despite the differences in affinity and desensitization characteristics between NMDA and AMPA receptors (Patneau and Mayer, 1990). Second, lowering the extracellular $\mathrm{Ca}^{2+} / \mathrm{Mg}^{2+}$ ratio attenuated PPD and tetanic depression. Most important, PPD and tetanic depression of EPSCs occurred without a decrease in the mean amplitude of mEPSCs. Any postsynaptic mechanism for depression, including glutamate receptor desensitization, would be expected to result in a decrease in the amplitude of mEPSCs.

Although the predominant localization of the depression seems to be presynaptic, a small contribution of postsynaptic glutamate receptor desensitization cannot be excluded. The KolmogorovSmirnov Test is a powerful and conservative tool for detecting a difference between two independent small samples of mEPSC amplitudes (Goodman, 1954; Siegel, 1956). When the Kolmogorov-Smirnov Test failed to detect a significant difference, we concluded that there was no difference between the two distributions or that the difference was too small to detect. When we estimated thresholds for detection with the Kolmogorov-Smirnov test, as described in Materials and Methods, the threshold varied from 5 to $20 \%$. Therefore it is possible that a relatively small postsynaptic contribution to PPD and TD would not be detected. Another possible source of error in our presynaptic localization of tetanic depression on the basis of the Kolmogorov-Smirnov test is that we cannot account for the contribution of mEPSC amplitudes occurring during the decay phase of EPSCs. However, two factors lessen our concern about this issue. First, given the high frequency of mEPSCs occurring for several hundred msec after a train of action potentials, the mEPSCs occurring during the first $40-50$ 
Table 2. Effect of $\mathrm{Cd}^{2+}(500 \mu \mathrm{M})$ on mEPSC charge distribution and frequency of occurrence

\begin{tabular}{lccccc} 
& \multicolumn{2}{l}{$\begin{array}{l}\text { Mean mEPSC charge } \\
\text { (picocoulombs) }\end{array}$} & & \multicolumn{2}{l}{$\begin{array}{l}\text { Mean interevent interval } \\
(\mathrm{msec})\end{array}$} \\
\cline { 2 - 3 } \cline { 5 - 6 } $\begin{array}{l}\text { Cell } \\
\text { number }\end{array}$ & Control & $\mathrm{Cd}^{2+}$ & & Control & $\mathrm{Cd}^{2+}$ \\
\hline 1 & $74 \pm 58$ & $62 \pm 49$ & & $503 \pm 522$ & $426 \pm 401$ \\
2 & $107 \pm 110$ & $128 \pm 132$ & & $2960 \pm 3090$ & $2974 \pm 2806$ \\
3 & $68 \pm 46$ & $81 \pm 68$ & & $542 \pm 509$ & $596 \pm 625$ \\
4 & $58 \pm 34$ & $58 \pm 32$ & & $2066 \pm 2184$ & $2159 \pm 2035$ \\
5 & $71 \pm 52$ & $79 \pm 50$ & & $4593 \pm 4991$ & $2877 \pm 3338^{a}$ \\
6 & $\mathrm{NA}^{b}$ & & & $142 \pm 131$ & $104 \pm 103^{a}$
\end{tabular}

${ }^{a}$ In these recordings, the mEPSC frequency of occurrence actually increased in $\mathrm{Cd}^{2+}$ $(p<0.05)$.

${ }^{b}$ In this recording, drift in postsynaptic membrane holding current precluded analysis of change in mEPSC charge distribution with $\mathrm{Cd}^{2+}$ application.

msec would be unlikely to affect significantly the post-train amplitude distribution constructed from repeated trials for each recording. Second, in contrast to other recent work examining the localization of PPD of EPSCs at excitatory synapses that used mEPSCs occurring up to $5 \mathrm{sec}$ after stimulation to construct poststimulus amplitude distributions (Mennerick and Zorumski, 1995), we limited the poststimulus amplitude distribution to those mEPSCs occurring $<1 \mathrm{sec}$ after the paired-pulse or tetanic stimuli.

Finally, in any experiment examining the behavior of mEPSCs, even at synapses between relatively isolated pairs of neurons, the possibility remains that some mEPSCs may arise from nerve terminals from neurons other than the monitored presynaptic cell and that these mEPSCs could shift the amplitude distributions for mEPSCs analyzed here. It is difficult to estimate the contribution of spontaneous mEPSCs to the sampled distribution of mEPSCs occurring before and after paired-pulse or tetanic stimulation. However, the ability to stimulate reliable paired-pulse facilitation of asynchronous release of mEPSCs suggests that a relatively stationary population of active zones participates in the asynchronous release activated by each action potential. We conclude that presynaptic mechanisms are sufficient to account for both PPD and tetanic depression. Therefore, presynaptic mechanisms for short-term synaptic plasticity must account for both a paired-pulse facilitation of mEPSC frequency and frequency-dependent depression of EPSCs.

\section{The role of $\mathrm{Ca}^{2+}$ in the tetanic increase in mEPSC frequency accompanying tetanic depression}

Several pieces of evidence suggest that the tetanic increase in mEPSC frequency is coupled to synaptic activity via $\mathrm{Ca}^{2+}$ accumulation in the nerve terminal: (1) lowering the extracellular $\mathrm{Ca}^{2+} / \mathrm{Mg}^{2+}$ ratio attenuates the increase in mEPSC frequency, (2) extracellular $\mathrm{Cd}^{2+}$ blocks the increase in mEPSC frequency accompanying presynaptic action potentials without disrupting spontaneous presynaptic neurotransmitter release or changing postsynaptic properties, and (3) membrane-permeant $\mathrm{Ca}^{2+}$ chelators attenuate the increase in mEPSC frequency.

Our results with $\mathrm{Ca}^{2+}$ chelators are limited to qualitative conclusions. Our confidence that calcium chelators are actually buffering $\mathrm{Ca}^{2+}$ instead of working through some other effect is based on four findings: (1) Two chelators with similar $\mathrm{Ca}^{2+}$ affinities $\left(K_{\mathrm{D}}\right.$; Tymianski et al., 1994) show similar attenuation of the mEPSC frequency increase. (2) The faster $\mathrm{Ca}^{2+}$ buffering ability (higher forward $\mathrm{Ca}^{2+}$ association rate constant, $K_{\text {on }}$ ) of
BAPTA-AM (Tymianski et al., 1994) correlates with the ability of BAPTA-AM to disrupt fast release and tetanic depression and the inability of EGTA-AM, a slower $\mathrm{Ca}^{2+}$ buffer, to affect fast release and tetanic depression. (3) The effects of both BAPTA-AM and EGTA-AM on the increase in mEPSC frequency can be reversed by longer trains of action potentials, suggesting that the effects of calcium chelators are saturable. (4) Our ability to resolve quantal amplitudes allowed us to ascertain that, in our preparation, BAPTA-AM exerts its effects primarily via presynaptic mechanisms. The difference between our results and previous results in hippocampal slice suggesting a significant postsynaptic effect may be the result of a difference in preparation or the more indirect methods of synaptic plasticity localization used in previous work (Niesen et al., 1991). We conclude that our experiments with BAPTA-AM demonstrate that calcium buffering in the nerve terminal can be manipulated rapidly and reliably at our hippocampal synapses in culture. The persistence of evoked neurotransmission and frequency-dependent depression in EGTA-AM with the attenuation of the accompanying facilitation of mEPSC frequency strongly suggests that facilitation of $\mathrm{mEPSC}$ frequency is a $\mathrm{Ca}^{2+}$-dependent process distinct from the $\mathrm{Ca}^{2+}$-dependent processes that may regulate evoked synchronous release and frequency-dependent depression of EPSCs.

\section{Calcium dependence of frequency-dependent depression of EPSCs}

Our experimental results provide two lines of evidence, which suggest that frequency-dependent depression of EPSCs occurs independent of the activity-dependent accumulation of calcium in the nerve terminal. Whereas lowering the extracellular $\mathrm{Ca}^{2+}$ / $\mathrm{Mg}^{2+}$ concentration ratio blocked the calcium accumulationdependent tetanic increase in mEPSC frequency, frequencydependent depression of EPSCs persisted, although at a somewhat attenuated level (see Fig. 1). Also, consistent with previous experiments at the squid giant synapse (Swandulla et al., 1991), calcium chelation with EGTA-AM, which selectively blocked facilitation of asynchronous release, had no significant effect on tetanic depression of EPSCs (see Fig. 8). In fact, frequency-dependent depression is more likely to be dependent on a process activated by a transient increase in $\mathrm{Ca}^{2+}$, which is unaffected by a slow chelator like EGTA-AM but blocked by the more rapid calcium chelator, BAPTA-AM (see Fig. 7). Other forms of short-term plasticity, including post-tetanic potentiation (PTP) at the crayfish neuromuscular junction (Kamiya and Zucker, 1994) and PTP at the hippocampal mossy fiber synapse recorded with low external $\mathrm{Ca}^{2+} / \mathrm{Mg}^{2+}$ concentration ratios (Regehr et al., 1994), depend on the time course of accumulation of $\mathrm{Ca}^{2+}$ in nerve terminal. The absence of PTP of EPSCs at excitatory hippocampal synapses in culture that show activity-dependent accumulation of calcium in the nerve terminal, indicated by the increase in asynchronous release of mEPSCs, may be a consequence of the relatively physiological external $\mathrm{Ca}^{2+} / \mathrm{Mg}^{2+}$ concentration ratios used in these experiments or the developmental state of the release properties of embryonic cultured hippocampal neurons (Muller et al., 1993, 1995; Bolshakov and Siegelbaum, 1995). It remains to be determined whether the transient peak increase in presynaptic nerve terminal $\mathrm{Ca}^{2+}$ concentration accompanying an action potential results in depression of EPSCs via depletion of vesicles available for synchronous release (Zucker, 1989) or whether the transient $\mathrm{Ca}^{2+}$ signal could activate an independent calcium-dependent "braking" mechanism for depression 


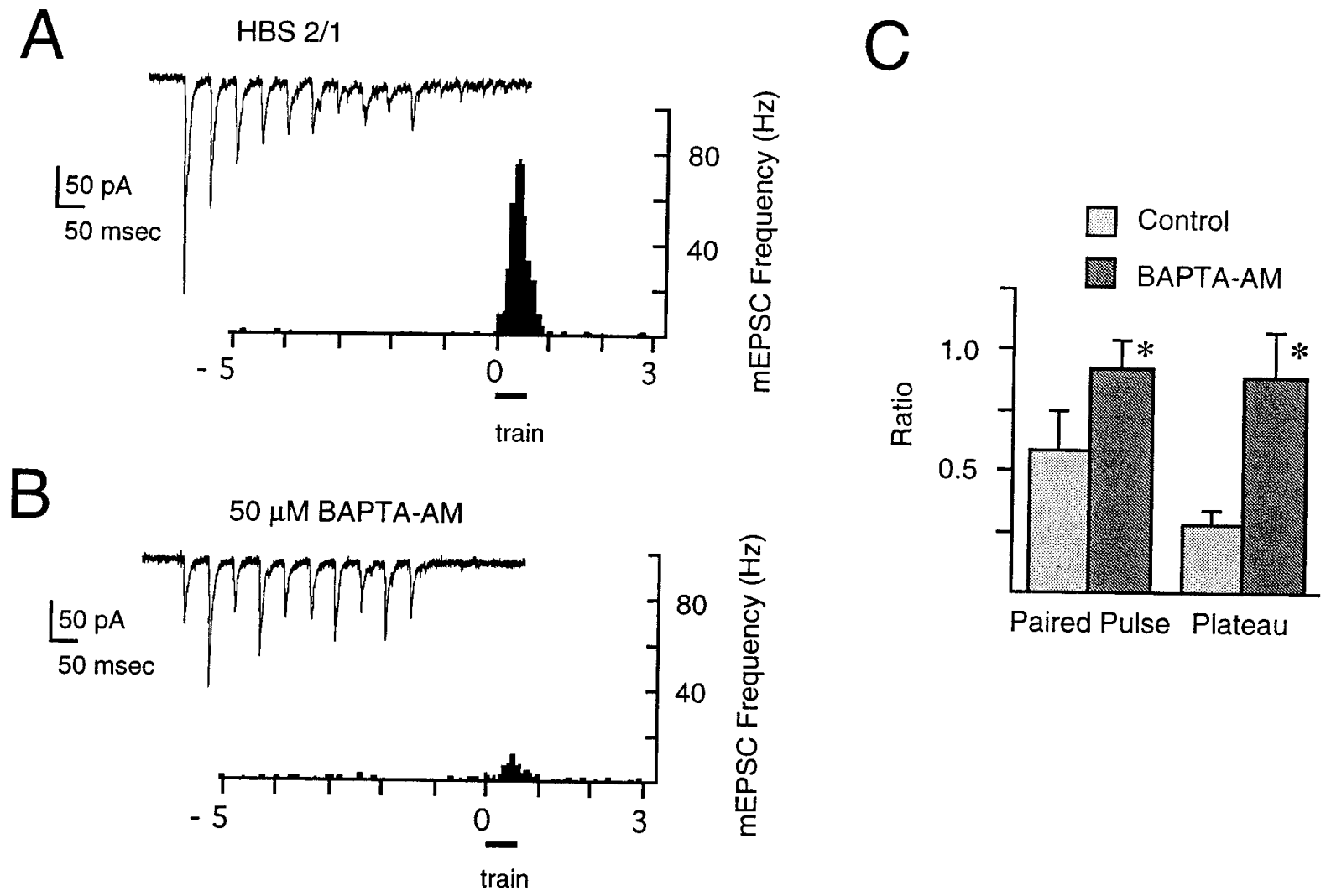

D

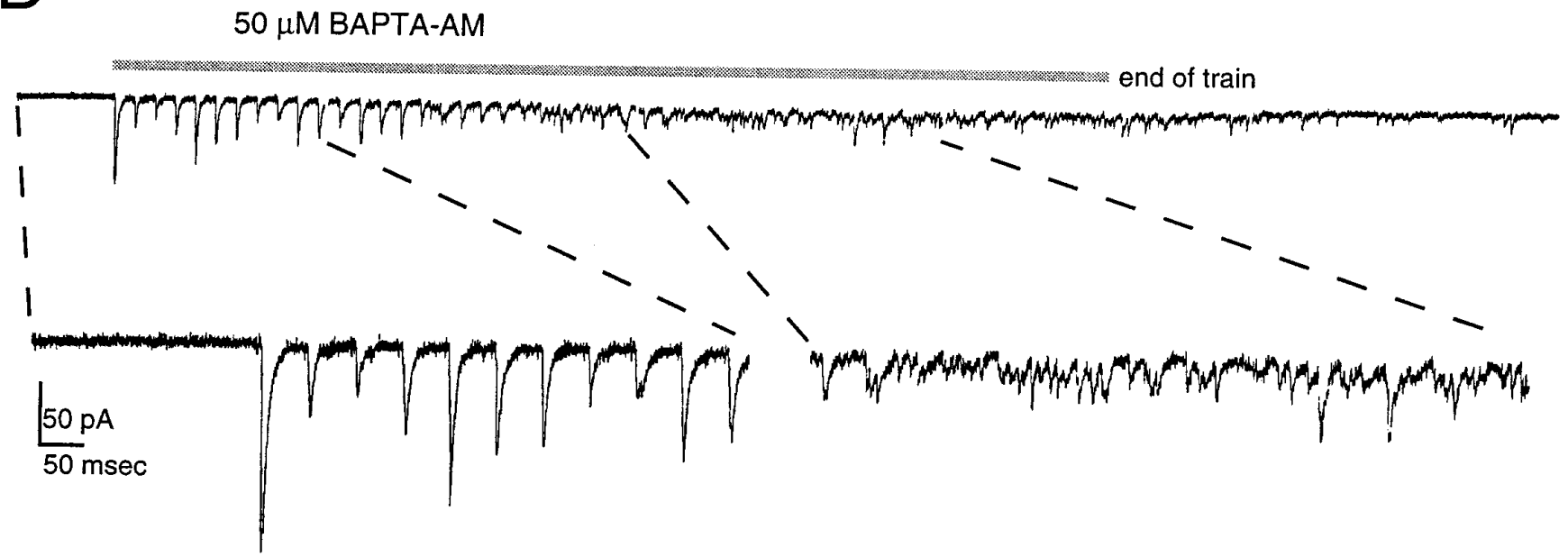

Figure 7. Calcium chelation blocks mEPSC frequency increase and attenuates frequency-dependent depression accompanying $20 \mathrm{~Hz}$ stimulation. $A$, In HBS 2:1, a representative trace shows tetanic depression of EPSCs and an increase in mEPSC frequency. The accompanying occurrence time-binned histogram plots the mean increase in the occurrence of mEPSCs during and after the evoked train of action potentials for nine trials. $B$, In the same recording in the presence of $50 \mu \mathrm{M}$ BAPTA-AM, tetanic depression is attenuated. The accompanying histogram demonstrates mean attenuation of mEPSC frequency increase for 21 trials. $C$, The effect of calcium chelation with $50 \mu \mathrm{M}$ BAPTA-AM on tetanic depression was determined by analyzing both the paired-pulse ratio and the plateau ratio for EPSCs induced by $20 \mathrm{~Hz}$ trains of presynaptic action potentials. Plot shows paired-pulse and plateau ratio in control conditions and with $50 \mu \mathrm{M}$ BAPTA-AM added. BAPTA-AM results in a significant attenuation of paired-pulse and tetanic depression as assessed by plateau ratio ( $p<0.05$ for two-tailed paired two-sample Student's $t$ test; $n=7)$. *, Significant difference. $D$, With continued perfusion of $50 \mu \mathrm{M}$ BAPTA-AM, a longer train stimulus (50 action potentials instead of 10 ) results in recovery of the mEPSC frequency increase.

of synchronous neurotransmitter release (Mori et al., 1994). However, the coincident paired-pulse facilitation of mEPSC frequency and PPD of EPSCs suggests that simple depletion of vesicles is unlikely to explain the behavior. Our results demon- strate that intraterminal $\mathrm{Ca}^{2+}$ homeostasis can be manipulated rapidly in subtle ways that will allow the importance of multiple Ca-dependent processes in short-term plasticity to be distinguished at a model CNS synapse. 


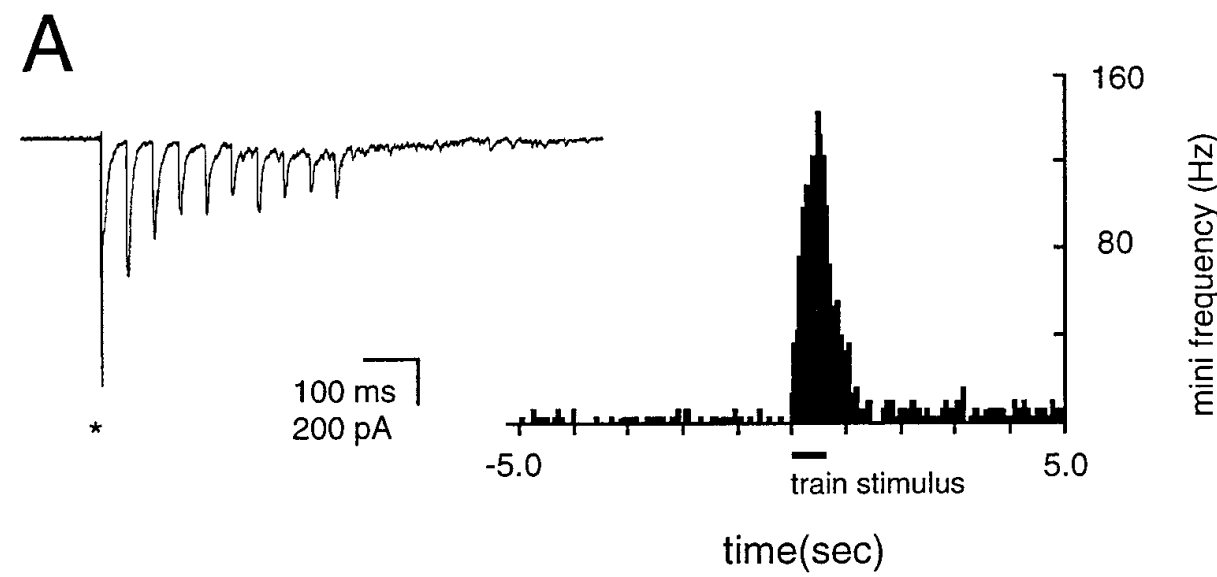

Figure 8. EGTA-AM eliminates tetanic increase in mEPSC frequency, but it does not affect tetanic depression of evoked EPSCs. $A$, Under control conditions, EPSCs that are evoked via trains of action potentials $(20 \mathrm{~Hz})$ show marked depression, whereas mEPSC release is increased. The histogram plots the mean increase in the occurrence of mEPSCs during and after the evoked train of action potentials (6 trials). *, EPSC amplitude value off scale. $B$, In the same recording, application of EGTA-AM $(50 \mu \mathrm{M})$ does not block the frequency-dependent depression of EPSCs. However, as the histogram demonstrates, the increase in mEPSC frequency that is observed during and after the train of action potentials, as in $A$, is blocked completely by the application of EGTA-AM (mean of 22 trials; bin size $=50 \mathrm{msec}$ ). Similar attenuation of mEPSC frequency increase was present in a total of five recordings. $C$, Graph summarizes behavior of the tetanic responses to $20 \mathrm{~Hz}$ trains of action potentials in control and 50$100 \mu \mathrm{M}$ EGTA-AM. Similar PPD and tetanic depression, as manifested in the plateau ratio, occurred in both conditions $(n=3)$. No significant difference was detected for either the paired-pulse or the plateau ratios $(p<0.05$; two-tailed paired two-sample Student's $t$ test). SD error bar is not visible for the paired-pulse ratio in control conditions, because the value was $<0.02$.
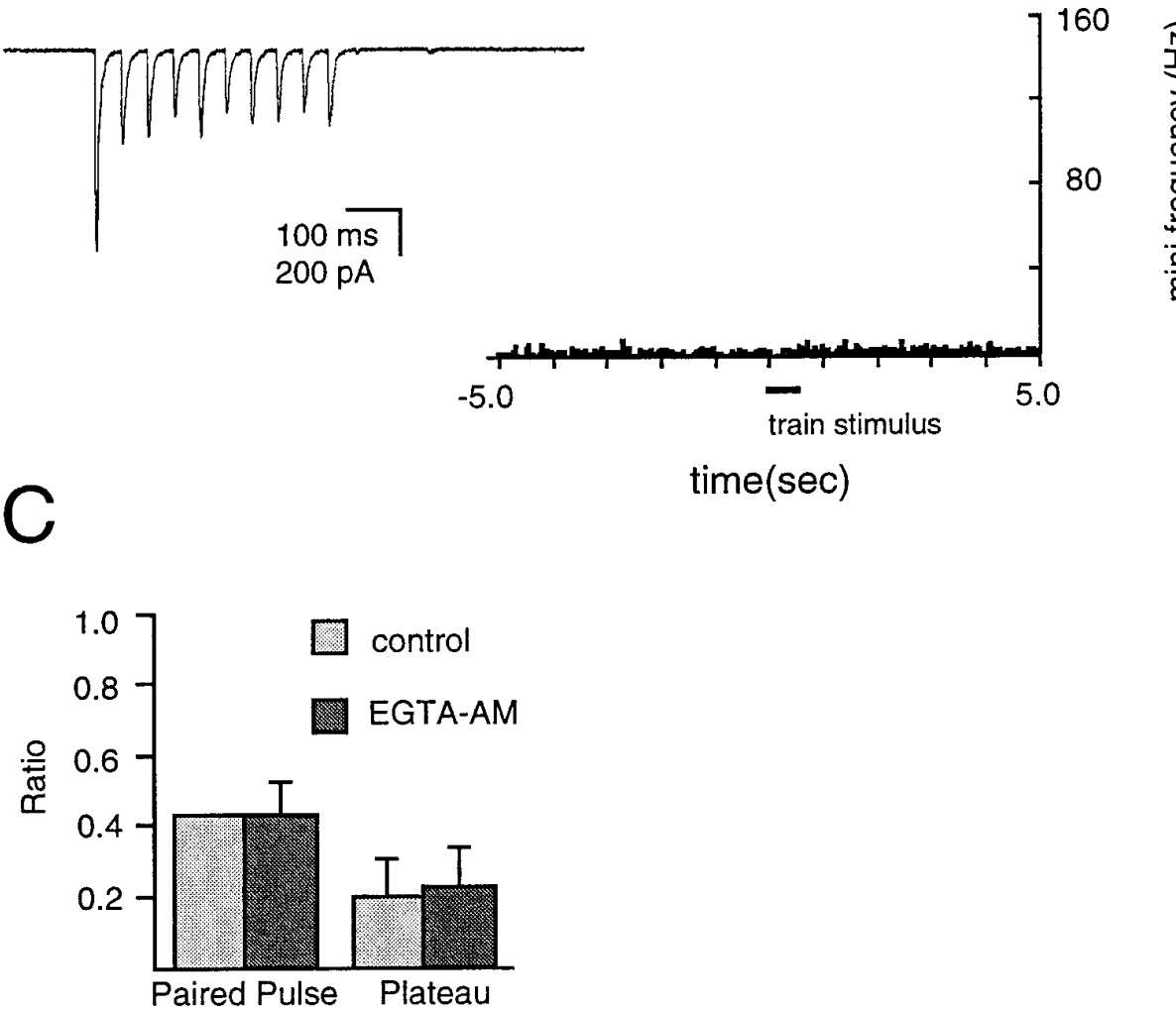

\section{REFERENCES}

Adler EM, Augustine GJ, Duffy SN, Charlton MP (1991) Alien intracellular calcium chelators attenuate neurotransmitter release at the squid giant synapse. J Neurosci 11:1496-1507.

Augustine GJ, Adler EM, Charlton MP (1991) The calcium signal for transmitter secretion from presynaptic nerve terminals. Ann NY Acad Sci 635:365-381.

Bolshakov VY, Siegelbaum SA (1995) Regulation of hippocampal transmitter release during development and long-term potentiation. Science 269:1730-1734.

Connors BW, Gutnick MJ (1990) Intrinsic firing patterns of diverse neocortical neurons. Trends Neurosci 13:99-104.

Cummings DD, Dichter MA (1994) Short-term plasticity of excitatory synapses between hippocampal neurons in dissociated cell culture. Soc Neurosci Abstr 20:1339.
Creager R, Dunwiddie T, Lynch G (1980) Paired-pulse and frequency facilitation in the CA1 region of the in vitro rat hippocampus. J Physiol (Lond) 299:409-424.

del Castillo J, Katz B (1954) Quantal components of the end-plate potential. J Physiol (Lond) 124:560-573.

Diamond JS, Jahr CE (1995) Asynchronous release of synaptic vesicles determines the time course of the AMPA receptor-mediated EPSC. Neuron 15:1097-1107.

Fatt P, Katz B (1952) Spontaneous subthreshold activity at motor nerve endings. J Physiol (Lond) 117:109-128.

Forsythe I, Clements J (1990) Presynaptic glutamate receptors depress excitatory monosynaptic transmission between mouse hippocampal neurones. J Physiol (Lond) 429:1-16.

Frankenhaeuser B, Hodgkin AL (1957) The action of calcium on the electrical properties of squid axons. J Physiol (Lond) 137:218-244. 
Geppert M, Goda Y, Hammer RE, Li C, Rosahl TW, Stevens CF, Sudhof TC (1994) Synaptotagmin I: a major $\mathrm{Ca}^{2+}$ sensor for transmitter release at a central synapse. Cell 79:717-727.

Goda Y, Stevens CF (1994) Two components of transmitter release at a central synapse. Proc Natl Acad Sci USA 91:12942-12946.

Goodman LA (1954) Kolmogorov-Smirnov tests for psychological research. Psychol Bull 51:160-168.

Griffith WH (1990) Voltage-clamp analysis of post-tetanic potentiation of the mossy fiber to CA3 synapse in hippocampus. J Neurophysiol 63:491-501.

Hamill OP, Marty A, Neher E, Sakmann B, Sigworth FJ (1981) Improved patch-clamp techniques for high-resolution current recording from cell and cell-free membrane patches. Pflügers Arch 391:85-100.

Kamiya H, Zucker RS (1994) Residual $\mathrm{Ca}^{2+}$ and short-term synaptic plasticity. Nature 371:603-606.

Katz B (1969) The release of neural transmitter substances. Liverpool: Liverpool UP.

Korn H, Faber DS (1991) Quantal analysis and synaptic efficacy in the CNS. Trends Neurosci 14:439-445.

Maki R, Cummings DD, Dichter MA (1995) Frequency-dependent depression of excitatory synaptic transmission is independent of activation of MCPG-sensitive presynaptic metabotropic glutamate receptors in cultured hippocampal neurons. J Neurophysiol 74:1671-1674.

Malenka RC, Nicoll RA (1993) NMDA-receptor-dependent synaptic plasticity: multiple forms and mechanisms. Trends Neurosci 16: 521-527.

Mallart A, Martin AR (1968) The relation between quantal content and facilitation at the neuromuscular junction of frog. J Physiol (Lond) 196:593-604.

McLarnon JG, Saint DA, Quastel DMJ (1986) The actions of dimethyl sulfoxide on neuromuscular transmission. Mol Pharmacol 30:631-638.

McNaughton BL (1980) Evidence for two physiologically distinct perforant pathways in the fascia dentata. Brain Res 199:1-19.

Mennerick S, Zorumski CF (1995) Paired-pulse modulation of fast excitatory synaptic currents in microcultures of rat hippocampal neurons. J Physiol (Lond) 488:85-101.

Mori A, Takahashi T, Miyashita Y, Kasai H (1994) Two distinct glutamatergic synaptic inputs to striatal medium spiny neurones of neonatal rats and paired-pulse depression. J Physiol (Lond) 476:217-228.

Muller D, Buchs PA, Stoppini L (1993) Time course of synaptic development in hippocampal organotypic cultures. Dev Brain Res 71:93-100.

Muller D, Oliver M, Lynch G (1995) Developmental changes in synaptic properties in hippocampus of neonatal rats. Dev Brain Res 49:105-114.
Niesen C, Charlton MP, Carlen PL (1991) Postsynaptic and presynaptic effects of the calcium chelator BAPTA on synaptic transmission in rat hippocampal dentate granule neurons. Brain Res 555:319-325.

Patneau D, Mayer M (1990) Structure-activity relationships for amino acid transmitter candidates acting at $N$-methyl-D-aspartate and quisqualate receptors. J Neurosci 10:2385-2399.

Rahamimoff R, Yaari Y (1973) Delayed release of transmitter at the frog neuromuscular junction. J Physiol (Lond) 228:241-257.

Regehr WG, Delaney KR, Tank DW (1994) The role of presynaptic calcium in short-term enhancement at the hippocampal mossy fiber synapse. J Neurosci 14:523-537.

Siegel S (1956) Nonparametric statistics for the behavioral sciences. New York: McGraw-Hill.

Stevens CF (1993) Quantal release of neurotransmitter and long-term potentiation. Cell [Suppl] 72:55-63.

Stevens CF, Wang Y (1995) Facilitation and depression at single central synapses. Neuron 14:795-802.

Swandulla D, Hans M, Zipser K, Augustine GJ (1991) Role of residual calcium in synaptic depression and post-tetanic potentiation: fast and slow calcium signaling in nerve terminals. Neuron 7:915-926.

Traub RD, Miles R (1991) Neuronal networks of the hippocampus. Cambridge: Cambridge UP.

Tymianski M, Wallace MC, Spigelman I, Masaaki U, Carlen PL, Tator $\mathrm{CH}$, Charlton MP (1993) Cell-permeant $\mathrm{Ca}^{2+}$ chelators reduce early excitotoxic and ischemic neuronal injury in vitro and in vivo. Neuron 11:221-235.

Tymianski M, Charlton MP, Carlen PL, Tator CH (1994) Properties of neuroprotective cell-permeant $\mathrm{Ca}^{2+}$ chelators: effects on $\left[\mathrm{Ca}^{2+}\right]_{\mathrm{i}}$ and glutamate neurotoxicity in vitro. J Neurophysiol 72:1973-1992.

Van der Kloot W (1991) The regulation of quantal size. Prog Neurobiol 36:93-130.

Wilcox KS, Dichter MA (1994) Paired-pulse depression in cultured hippocampal neurons is due to a presynaptic mechanism independent of $\mathrm{GABA}_{\mathrm{B}}$ autoreceptor activation. J Neurosci 14:1775-1788.

Wilcox KS, Buchhalter J, Dichter MA (1994) Properties of inhibitory and excitatory synapses between hippocampal neurons in very low-density cultures. Synapse 18:128-151.

Zengel JE, Sosa MA (1994) Changes in MEPP frequency during depression of evoked release at the frog neuromuscular junction. J Physiol (Lond) 477:267-277.

Zhang S, Trussell LO (1994) Voltage-clamp analysis of excitatory synaptic transmission in the avian nucleus magnocellularis. J Physiol (Lond) 480:123-136.

Zucker RS (1989) Short-term synaptic plasticity. Annu Rev Neurosci 12:13-31. 